\title{
Análise isocinética da articulação do cotovelo em crianças com paralisia cerebral ${ }^{1}$
}

\author{
Patrícia Martins Franciulli ${ }^{1,2}$ \\ Flávia de Andrade e Souza ${ }^{2}$ \\ Aline Bigongiari ${ }^{1,2}$ \\ Luis Mochizuki $^{1}$ \\ Rubens Correa Araújo ${ }^{3}$
}

${ }^{1}$ Laboratório de Biomecânica, Escola de Educação Física e Esporte, Universidade de São Paulo, SP, Brasil

${ }^{2}$ Laboratório de Biomecânica, Universidade São Judas Tadeu, São Paulo, SP, Brasil

${ }^{3}$ Departamento de Fisioterapia, Universidade de Taubaté, SP, Brasil

\begin{abstract}
Resumo: A quantidade de torque aplicado na articulação é uma medida de aptidão física importante para crianças com paralisia cerebral. O presente estudo analisou parâmetros cinéticos na articulação do cotovelo em crianças saudáveis e com paralisia cerebral. Participaram 10 crianças com paralisia cerebral e 10 crianças sem comprometimento neurológico. Avaliou-se a média do pico de torque, média do ângulo do pico de torque, coeficiente de variação do torque e aceleração angular do movimento de flexo-extensão do cotovelo nas velocidades com um dinamômetro isocinético. A média de pico de torque (extensão), aceleração (flexão) e coeficiente de variação (flexão e extensão) são diferentes entre grupos. Conclui-se que o torque e aceleração sofreram interferências no movimento de flexo-extensão; as principais diferenças encontradas foram entre os extremos das velocidades; não houve diferenças no ângulo do pico de torque. A espasticidade não interferiu na força dos músculos agonistas do movimento de flexão da articulação do cotovelo.
\end{abstract}

Palavras chaves: Paralisia Cerebral. Biomecânica. Torque.

\section{Isokinetic analysis of the elbow joint in children with cerebral palsy}

\begin{abstract}
The joint torque is an important variable related to children with cerebral palsy. The present study analyzed kinetic parameters during elbow flexion and extension movements in healthy and cerebral palsy children. Ten healthy and 10 cerebral palsy children participated of the study. An isokinetic dynamometer was used to measure the elbow mean peak torque, mean angle peak torque, coefficient of variation and acceleration during flexion and extension movements at different angular speeds. The mean peak torque on extension movement in healthy children group was significant higher compared to the cerebral palsy group. The coefficient of variation on both flexion and extension movements was significantly higher in cerebral palsy group. However there were significantly difference on both groups compared the lowest and highest velocities. Although the results showed no difference in flexor peak torque, the acceleration is significantly lower in lowest and highest angular velocity.
\end{abstract}

Key words: Cerebral Palsy. Biomechanics. Torque.

\section{Introdução}

As crianças com paralisia cerebral (PC) apresentam problemas no controle motor e postural, como as disfunções sensório-motoras e menor equilíbrio, e na aptidão física, como as alterações no tônus e força muscular (LANFREDI et al., 2004; MANCINI et al., 2002). Esses problemas afetam a execução de tarefas da vida diária. Para a adequada abordagem clínica para 0 tratamento dessas crianças é importante a

\footnotetext{
1 Este trabalho foi apresentado parcialmente no XIII Congresso Brasileiro de Biomecânica, realizado na FAU-USP, organizado pela EEFEUSP em 28 de julho à 01 de agosto de 2009.
}

avaliação dessas disfunções sensório-motoras e dos déficits na aptidão física.

A avaliação da força muscular em pessoas com disfunções motoras pode ser feita por meio de teste manual; contudo, o resultado não é satisfatório (AYALON et al, 2000) porque essas medidas manuais se limitam a alguns grupos musculares e são capazes apenas de obter uma medida discreta da força máxima. Por outro lado, o dinamômetro isocinético permite a avaliação da ação muscular no desempenho de movimentos monoarticulares em várias velocidades, mensurando o torque, trabalho e a relação líquida dos músculos agonista/antagonista 
(BITTENCOURT et al., 2005) de uma articulação. Essas medidas podem ser realizadas para 0 acompanhamento e diagnóstico individual (SCHNEIDER, RODRIGUES \& MEYER, 2002). Assim, o dinamômetro isocinético pode ser usado em crianças com PC para o adequado de diagnóstico e identificação de necessidades para um plano de tratamento específico (DURIGON,

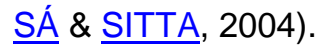

Desta forma, crianças com PC são capazes de produzir a mesma quantidade de torque articular que uma criança sem esse problema realiza? Para responder essa questão, o objetivo deste estudo foi comparar entre crianças saudáveis e com PC do tipo diparesia espástica os parâmetros cinéticos durante o movimento de flexo-extensão da articulação do cotovelo no membro dominante.

Devido às alterações sensório-motoras das crianças com PC, propomos como hipótese deste estudo que o torque máximo na articulação do cotovelo de crianças com PC é menor do que o gerado por crianças sem PC.

\section{Método}

\section{Participantes}

Vinte crianças foram distribuídas nos grupos GSA (10 crianças sem comprometimento neurológico, $8,2 \pm 1,9$ anos; $130,7 \pm 10,2 \mathrm{~cm}$ e $28,7 \pm 7,0 \mathrm{~kg}$ ) e GPC (10 crianças com PC, 8,6 \pm
1,5 anos; $122,0 \pm 9,9 \mathrm{~cm}$ e $23,8 \pm 9,6 \mathrm{~kg}$ ). Os critérios de inclusão para GSA foram ter idade entre 6-12 anos, ambos os sexos, ausência de comprometimento neurológico, enquanto que os critérios de exclusão foram cirurgias recentes nos últimos 6 meses. Os critérios de inclusão para a composição do GPC foram ter idade entre 6-12 anos, ambos os sexos, PC do tipo diparesia espástica; e os critérios de exclusão foram PC do tipo diparesia espástica com alterações cognitivas e/ou mista (atetoses e ataxias), alterações visuais, deformidades instaladas, cirurgias recentes em membros superiores e/ou uso de fenol e toxina botulínica (últimos 6 meses).

\section{Procedimentos}

Este estudo foi aprovado pelo Comitê de Ética em Pesquisa local (protocolo 024/2008). Antes do experimento, os responsáveis pelas crianças assinaram um termo de consentimento livre e esclarecido.

O teste de dominância lateral de Edimburgo foi usado para determinar o membro dominante; do qual foram utilizados os itens desenhar, arremessar, uso de tesoura, escovar os dentes, uso de colher e abrir uma caixa.

O grau de espasticidade dos grupos musculares envolvidos no momento analisado das crianças com PC foi avaliado por meio da escala de Ashworth Modificada por um único avaliador (Tabela 1).

Tabela 1. Classificação da espasticidade nos membros superiores das crianças do GPC segundo a escala de Ashworth modificada, realizado por um único avaliador.

\begin{tabular}{|c|c|c|c|c|c|c|c|c|c|c|c|c|}
\hline \multirow[b]{3}{*}{ Sujeito } & \multicolumn{4}{|c|}{ Ombro } & \multicolumn{4}{|c|}{ Cotovelo } & \multicolumn{4}{|c|}{ Punho } \\
\hline & \multicolumn{2}{|c|}{ Flexor } & \multicolumn{2}{|c|}{ Extensor } & \multicolumn{2}{|c|}{ Flexor } & \multicolumn{2}{|c|}{ Extensor } & \multicolumn{2}{|c|}{ Flexor } & \multicolumn{2}{|c|}{ Extensor } \\
\hline & Dir & Esq & Dir & Esq & Dir & Esq & Dir & Esq & Dir & Esq & Dir & Esq \\
\hline 1 & 1 & 0 & 0 & 0 & 2 & 2 & 0 & 0 & 0 & 3 & 0 & 0 \\
\hline 2 & 0 & 0 & 0 & 0 & 0 & 2 & 2 & 0 & 1 & 1 & 0 & 0 \\
\hline 3 & 0 & 2 & 0 & 3 & 0 & 3 & 0 & 0 & 0 & 2 & 0 & 0 \\
\hline 4 & 2 & 1 & 0 & 3 & 2 & 3 & 0 & 0 & 2 & 2 & 0 & 0 \\
\hline 5 & 0 & 0 & 1 & 0 & 0 & 0 & 0 & 0 & 1 & 0 & 0 & 0 \\
\hline 6 & 0 & 1 & 0 & 0 & 0 & 0 & 0 & 0 & 2 & 0 & 0 & 0 \\
\hline 7 & 2 & 1 & 1 & 0 & 2 & 1 & 0 & 0 & 0 & 2 & 1 & 0 \\
\hline 8 & 1 & 1 & 1 & 0 & 1 & 0 & 0 & 0 & 0 & 0 & 0 & 1 \\
\hline 9 & 1 & 1 & 0 & 0 & 2 & 2 & 0 & 0 & 1 & 1 & 0 & 0 \\
\hline 10 & 0 & 0 & 1 & 1 & 0 & 0 & 0 & 0 & 0 & 0 & 0 & 0 \\
\hline mediana & 0,5 & 1 & 0 & 0 & 0,5 & 1,5 & 0 & 0 & 0,5 & 1 & 0 & 0 \\
\hline moda & 0 & 1 & 0 & 0 & 0 & 0 & 0 & 0 & 0 & 0 & 0 & 0 \\
\hline
\end{tabular}

As crianças foram acomodadas e atadas na cadeira do dinamômetro isocinético (Biodex System $3 \AA$ ) por meio de cintos no tronco e abdome para prevenir movimentos acessórios que modificassem o torque avaliado. $O$ eixo do braço de alavanca do dinamômetro foi alinhado com o epicôndilo lateral do cotovelo e, após esta 56 preparação, as crianças realizaram movimentos de flexo-extensão por 5 minutos para permitir a adaptação à máquina e à especificidade da tarefa.

O protocolo de movimentos no dinamômetro isocinético consistiu de quatro séries nas velocidades $30,60,90$ e $120 \%$ s e com 5 
repetições em cada velocidade (ROSS \& ENGSBERG, 2002), no modo isocinético para contrações concêntrica-concêntrica. A ordem das velocidades foi sorteada. Entre cada série houve um intervalo de $30 \mathrm{~s}$ e entre cada mudança de velocidade, um tempo de repouso de $180 \mathrm{~s}$. Para cada participante, foi ajustada as posições de máxima extensão e flexão do cotovelo. Esses ajustes determinaram a amplitude total de movimento durante $o$ teste $\left(0-140^{\circ}\right)$.

O torque líquido produzido no teste foi adquirido pelo software Biodex Medical System ${ }^{\circledR}$ e armazenado em PC Pentium para posterior análise.

\section{Variáveis analisadas}

A partir das séries temporais do torque foram calculados os seguintes parâmetros: pico de torque, posição angular da articulação do cotovelo no instante do pico de torque, coeficiente de variação do torque e aceleração angular de movimento. Cada uma dessas variáveis foi apresentada na fase de flexão e extensão do cotovelo. Essas variáveis foram obtidas no software, que gerencia o funcionamento do

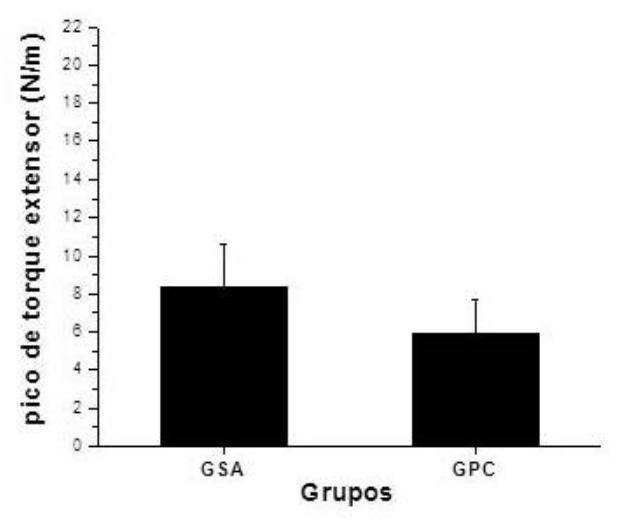

equipamento.

\section{Análise Estatística}

As médias do pico de torque, ângulo do pico de torque, aceleração angular e coeficiente de variação do torque foram comparadas por meio de análises de variância (ANOVA) de 2 fatores (grupo: GSA e GPC, e velocidade: $30,60,90$ e $120 \%$ s) e teste post hoc Tukey HSD $(p<0,05)$ foi usado para identificar as diferenças entre os níveis dos fatores. Esses testes estatísticos foram realizados no software Statistica (Statsoft, Inc, Tulsa, OK USA) e no software Origin 7.0.

\section{Resultados}

O pico de torque concêntrico na extensão do cotovelo sofreu efeito do grupo $\left(F_{(1,72)}=4,3\right.$ $p=0,04)$ e da velocidade $\left(F_{(3,72)}=3,0 p=0,03\right)$. $O$ pico de torque foi maior no GSA e na velocidade 30\% (Figura 1).

$O$ pico de torque concêntrico de flexão do cotovelo não sofreu efeito do grupo $\left(F_{(1,72)}=1,77\right.$ $\mathrm{p}=0,19)$ e sofreu efeito da velocidade $\left(\mathrm{F}_{(3,72)}=2,4\right.$ $p=0,04$ ) (Figura 2). O pico de torque foi maior na velocidade $30 \%$ s.

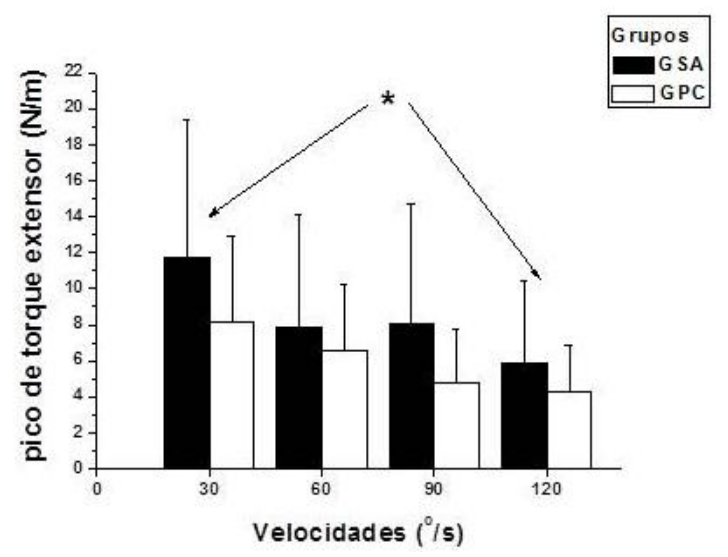

Figura 1. Média e desvio padrão do pico de torque extensor de cotovelo nos grupos GSA e GPC e média e desvio padrão do pico de torque das crianças dos grupos GSA e GPC divididos nas velocidades 30, 60, 90 e $120 \%$ s. * diferença estatisticamente significante entre 30 e $120 \%$ s.
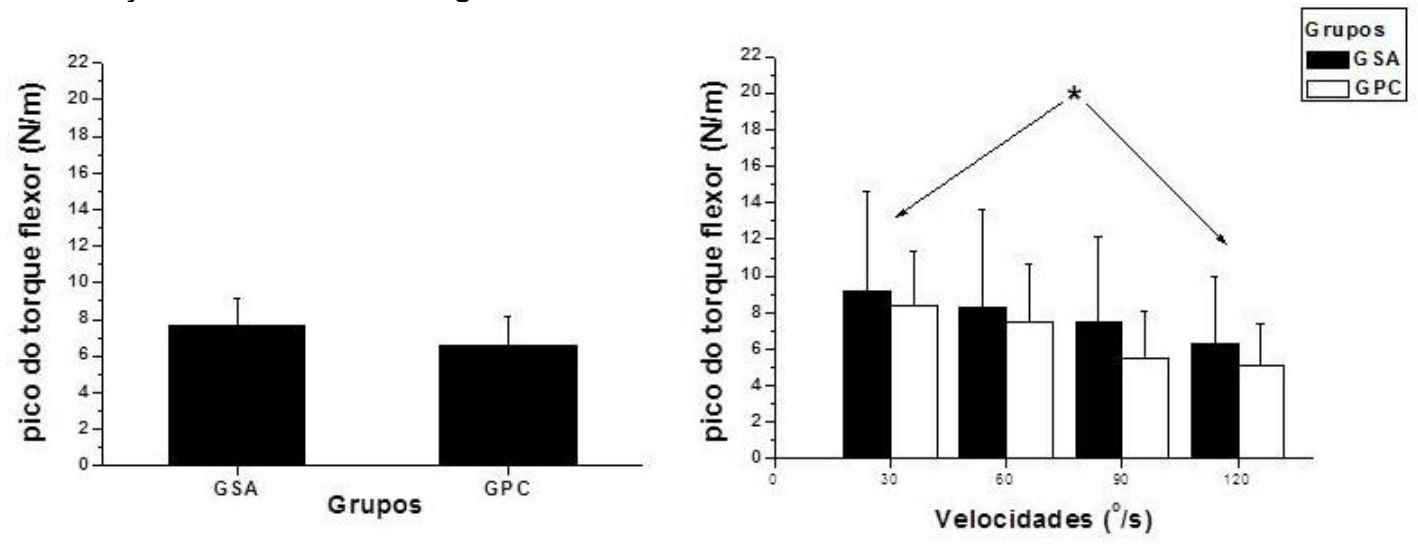

Figura 2. Média e desvio padrão do pico de torque flexor de cotovelo nos grupos GSA e GPC e média e desvio padrão do pico de torque das velocidades 30,60, 90 e $120 \%$. * diferença estatisticamente significante entre 30 e $120 \%$ s. 
Não houve efeito do grupo $\left(F_{(1,72)}=2,5 p=0,11\right)$ e velocidade $\left(F_{(3,72)}=2,2 p=0,09\right)$ no ângulo do pico de torque no movimento de extensão do cotovelo (Figura 3). $O$ ângulo do pico de torque no movimento de flexão do cotovelo não sofreu efeito do grupo $\left(F_{(1,72)}=3,2 \quad p=0,08\right)$ e nem da velocidade $\left(F_{(3,72)}=0,4 p=0,70\right)$ como pode ser visualizado na Figura 4.

A aceleração angular do movimento de extensão não sofreu efeito do grupo $\left(F_{(1,72)}=3,3\right.$ $p=0,07)$ e sofreu efeito da velocidade $\left(F_{(3,72)}=9,1\right.$ $p<0,001$ ) (Figura 5). A aceleração angular foi mais alta foi $120 \%$ s.
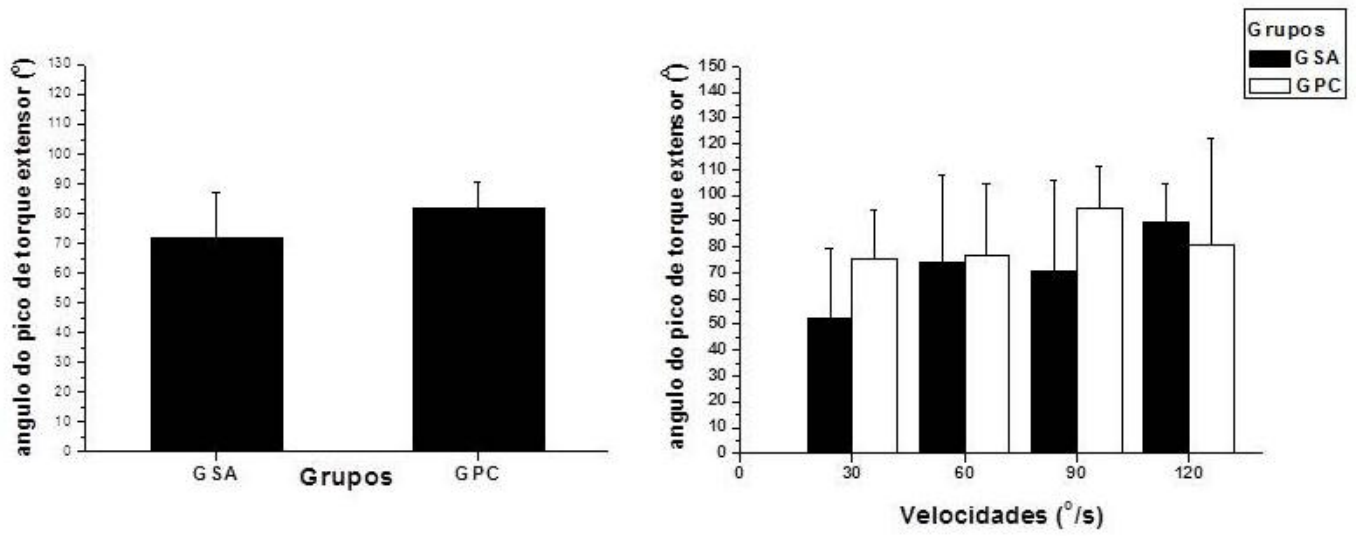

Figura 3. Média e desvio padrão do ângulo do pico de torque extensor de cotovelo nos grupos GSA e GPC e média e desvio padrão do pico de torque das velocidades $30,60,90$ e $120 \%$ s.
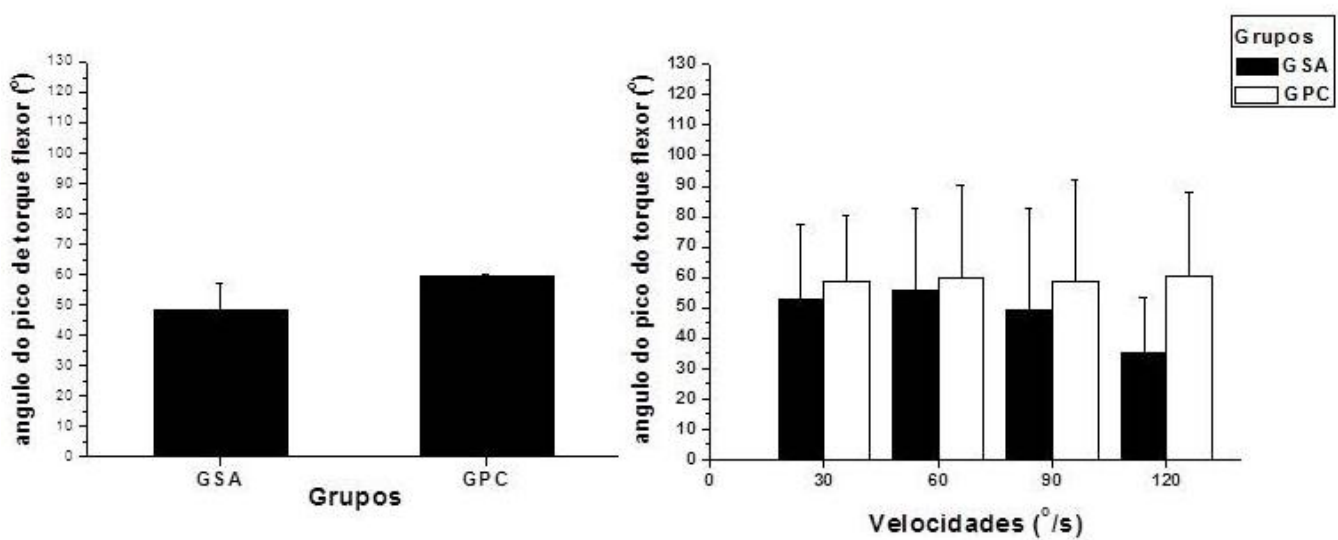

Figura 4. Média e desvio padrão do ângulo do pico de torque flexor de cotovelo nos grupos GSA e GPC e média e desvio padrão do pico de torque das velocidades 30, 60, 90 e $120 \%$ s.
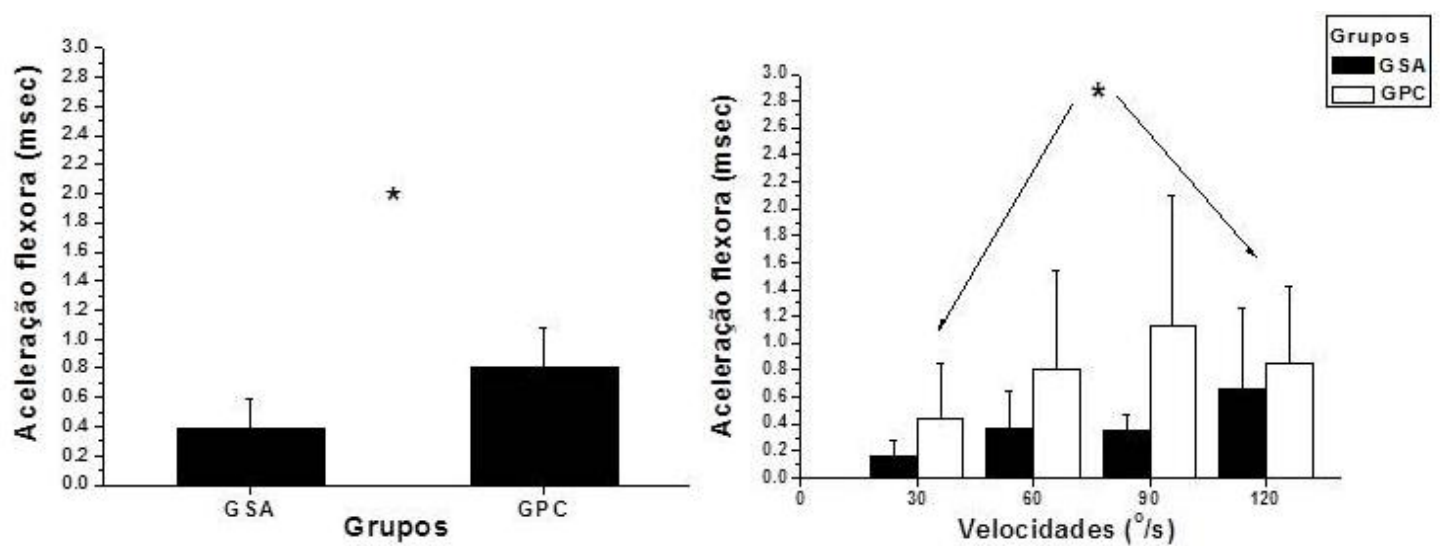

Figura 5. Média e desvio padrão da aceleração flexora nos grupos GSA e GPC e média e desvio padrão da aceleração $30,60,90$ e $120 \%$ s. * diferença estatisticamente significante entre grupos e velocidades 30 e $120 \%$ s.

A aceleração angular do movimento de flexão do cotovelo sofreu efeito do grupo $\left(F_{(1,72)}=11,4\right.$ $p<0,001)$ e da velocidade $\left(F_{(3,72)}=2,8 \quad p=0,04\right)$ (Figura 6). A aceleração angular do movimento de flexão foi maior no grupo GPC e $120 \%$ s. 
O CV do torque no movimento de extensão do cotovelo sofreu efeito do grupo $\left(F_{(1,72)}=6,2\right.$ $\mathrm{p}=0,01)$. O maior CV foi realizado no GPC. O CV do torque no movimento de extensão do cotovelo não sofreu efeito da velocidade $\left(F_{(3,72)}=1,93 p=\right.$ 0,13) (Figura 7).
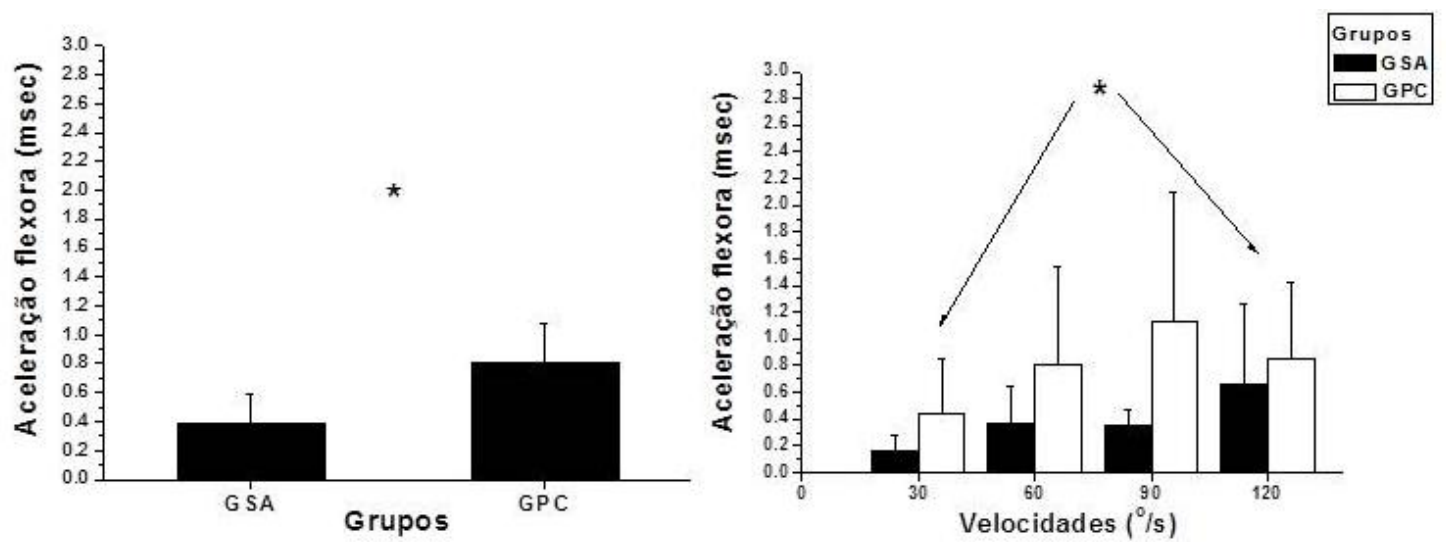

Figura 6. Média e desvio padrão da aceleração flexora nos grupos GSA e GPC e média e desvio padrão da aceleração 30,60, 90 e 120\%. * diferença estatisticamente significante entre grupos e velocidades 30 e $120 \%$ s.
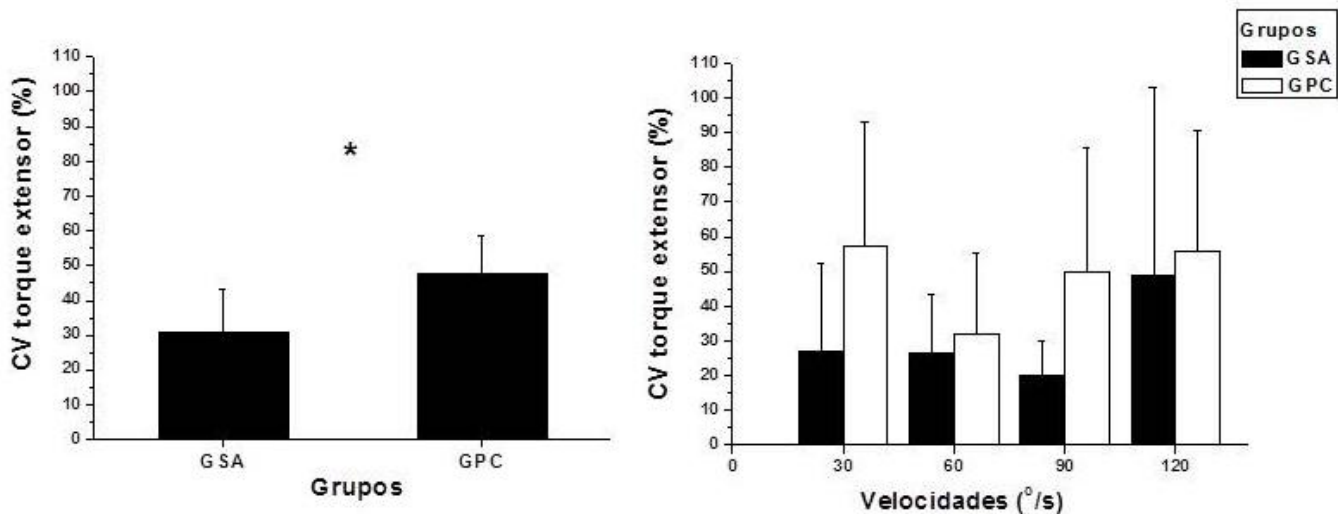

Figura 7. Média e desvio padrão do coeficiente de variação do torque extensor nos grupos GSA e GPC e média e desvio padrão do coeficiente de variação do torque nas velocidades $30,60,90$ e $120^{\circ} / \mathrm{s}$. * diferença estatisticamente significante entre grupos.
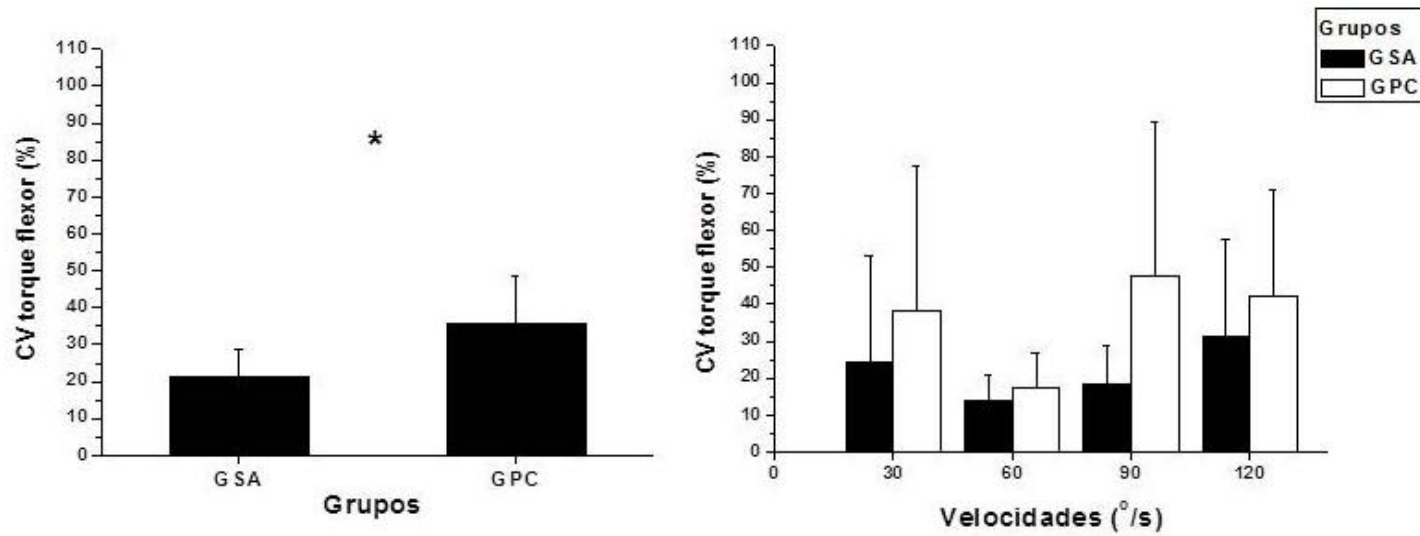

Figura 8. Média e desvio padrão do coeficiente de variação do torque flexor nos grupos GSA e GPC e média e desvio padrão do coeficiente de variação do torque nas velocidades $30,60,90$ e $120 \%$ s. * diferença estatisticamente significante entre grupos.

\section{Discussão}

O levantamento bibliográfico indicou que a maior parte dos estudos que mensuraram 0 torque isocinético em crianças com PC foi feita com 0 foco nos membros inferiores. Os resultados deste estudo ampliam a disponibilidade 
de informações, mostrando como se comporta 0 torque isocinético nos membros superiores dessa população. Verificamos que as crianças com PC apresentaram um pico médio de torque menor na extensão de cotovelo em comparação às crianças do grupo controle, como foi observado em DAMIANO et al. (2001).

Segundo DAMIANO et al. (2001), o torque resultante voluntário de crianças com PC espástica é menor, principalmente nos músculos antagonistas ao espásticos, em relação às crianças sem comprometimento neurológico. A diminuição do torque resultante no antagonista do músculo espástico é devido à hipertonia se intensificar com as emoções e durante movimentos voluntários, quando podem ocorrer co-contrações de músculos agonistas e antagonistas, o que prejudica a função dos membros acometidos (MILLER, 2002).

$\mathrm{Na}$ flexão de cotovelo, inesperadamente, as crianças com PC desenvolveram o mesmo pico de torque que as crianças do grupo controle. ROSS \& ENGSBERG (2002) enfatizam que as crianças com PC apresentam músculos mais fracos que os indivíduos sem comprometimento neurológico. Essa semelhança pode ter sido devido ao fato que as crianças com PC eram diparéticas espásticas com leve comprometimento de membros superiores. Além disto, segundo SCHOLTES et al (2008), a fraqueza muscular causa mais limitações na função motora de crianças com PC do que a espasticidade.

Os resultados indicam que o pico de torque e a aceleração são afetados pela velocidade de movimento. A média de pico de torque e aceleração (extensão e flexão de cotovelo) apresentam diferenças entre as velocidades de $30 \%$ e $120 \%$ s. TUZSON, GRANATA \& ABEL (2003) afirmam que a espasticidade é velocidade dependente, e que, a velocidade limiar espástica está relacionada às limitações na velocidade angular articular durante os movimentos e a performance funcional dos membros espásticos. Além disso, as diferenças provocadas pela espasticidade podem ser mais destacadas em velocidades de movimento mais altas (DAMIANO et al, 2002).

A avaliação do ângulo do pico de torque faz inferências sobre a curva comprimento-tensão e indiretamente fornece informações do comprimento muscular, ou seja, do alongamento 60 ou estiramento do músculo. Esta avaliação pode auxiliar o entendimento do efeito das intervenções terapêuticas. De acordo com AQUINO et al (2007), o torque produzido ao longo da amplitude de movimento articular pode ser influenciado pelo sistema músculo-esquelético e determinado pela variação do braço de alavanca à medida que ocorre um deslocamento articular e também pela relação comprimento-tensão do músculo.

Crianças com PC não aparentam ter alterações na relação tensão muscular máxima $\mathrm{e}$ posição angular. Não houve diferença do ângulo onde ocorreu o pico de torque de extensão e flexão em nossos resultados entre GSA e GPC. ENGSBERG et al (1996) mostraram que o ângulo do pico de torque de crianças $P C$ não variou em nenhuma velocidade de movimento. Somente foram encontradas diferenças no pico de torque quando as posições angulares eram diferentes (ENGSBERG et al, 1996). Essa semelhança acontece porque a medida do ângulo de pico de torque não separa o componente ativo e passivo durante o movimento, o que pode minimizar a variação, mas não significa que o valor é normal ou que não haja alteração de alongamento ou estiramento muscular, comparando o GSA e GPC.

A ausência de variação do ângulo do pico de torque pode ser explicada (AQUINO et al, 2007) pelas seguintes razões: a) componentes ativos e passivos dos músculos durante o movimento (diminuição de um ou aumento do outro), b) deslocamento da curva na direção de um maior comprimento-tensão que podem influenciar na mensuração do ângulo do pico de torque.

Esperávamos encontrar maior dificuldade na iniciação do movimento de extensão, devido à espasticidade flexora no membro superior, que impede o início e realização deste movimento. Uma característica dos músculos espásticos é a baixa mobilidade funcional e resistência muscular (MOREAU et al., 2008). Apesar disso, observamos que as crianças com PC não mostraram dificuldade para iniciar a tarefa, apesar de apresentarem maior aceleração de movimento que as crianças do grupo controle.

A diferença do $\mathrm{CV}$ do torque entre grupos foi semelhante à DROUIN et al (2003). O CV do torque entre as diferentes classes, com e sem comprometimento neurológico, pode ser encontrado dependendo do ângulo $X$ velocidade. AYALON et al (2000) relatam que os valores do 
CV do torque de extensão e flexão da articulação do joelho de crianças com PC são mais elevados quando comparados com crianças sem comprometimento neurológico. Esses dados, mesmo que analisados em articulações diferentes concordam com os valores no presente estudo, onde também foram encontrados valores maiores em crianças com PC.

Não encontramos diferenças entre as diferentes velocidades angulares, o que também pode ser observado em BORGES et al (2007), no qual a variabilidade na resposta no $\mathrm{CV}$ do torque não diferiu entre as posições testadas nas velocidades de $30 \%$ e $120 \%$ s, no movimento de supinação do antebraço.

O entendimento das variáveis analisadas permite direcionar e diversificar um programa de reabilitação para melhorar a atuação terapêutica do paciente com PC. Não houve diferença entre grupos para a maioria das variáveis. Essa observação permite adequar a intervenção terapêutica para as crianças com PC para uma melhor qualidade de vida, com base em avanços motores e a influência nos domínios cognitivos, interação social e independência.

\section{Referências}

AQUINO, C. F.; FREIRE, M. T. F.; NEVES, N. M.; FERREIRA, P. C. A.; FONSECA, S. T. Análise da confiabilidade de um método de mensuração do ângulo do pico de torque ativo dos isquiosssurais. Revista Brasileira de Fisioterapia, São Carlos, v. 11, p. $169-175,2007$

AYALON, M.; BEN-SIRA, D.; HUTZLER, Y.; GILAD, T. Reliability of isokinetic strength measurements of the knee in children with cerebral palsy. Developmental Medicine \& Child Neurology, London, v. 42, p. 398-402, 2000. Disponível em: <http://dx.doi.org/10.1017/S0012162200000724>. Acesso em: 9 nov. 2011

BITTENCOURT, N. F. N.; AMARAL, G. M.; ANJOS, M. T. S.; D'ALESSANDRO, R.; AURÉLIO, S. A.; FONSECA, S. T. Avaliação muscular isocinética da articulação do joelho em atletas das seleções brasileiras infanto e juvenil de voleibol masculino. Revista Brasileira de Medicina do Esporte, São Paulo, v. 11, p. 331336, 2005. Disponível em: $<$ http://dx.doi.org/10.1590/S151786922005000600005>. Acesso em: 9 nov. 2011

BORGES, K. C.; RODRIGUES, A. M.; LOSS, J. F.; PETERSEN, R. D. S.; OLIVEIRA, M. A. Efeito da posição da articulação do cotovelo no controle de torque de supinação do antebraço em jovens adultos. Revista Brasileira de Fisioterapia, São Carlos, v. 11, p. 487-493, 2007. Disponível em: $<$ http://dx.doi.org/10.1590/S1413-

35552007000600011 >. Acesso em: 9 nov. 2011.

DAMIANO, D. L.; QUINLIVAN, J.; OWEN, B. F.; SHAFFREY, M.; ABEL, M. F. Spasticity versus strength in cerebral palsy: relationships among involuntary resistance, voluntary torque, and motor function. European Journal of Neurology, Oxford, v.8, supl. 5, p.40-49, 2001. Disponível em: $<$ http://link.periodicos.capes.gov.br.ez87.periodico s.capes.gov.br/sfxlcl3?url ver $=$ Z39.88-

2004\&url ctx fmt=infofi/fmt:kev:mtx:ctx\&ctx enc= info:ofi/enc:UTF-8\&ctx ver=Z39.88-

2004\&rfr id=info:sid/sfxit.com:azlist\&sfx.ignore da te threshold=1\&rft.object id=954925617087\&svc. fulltext=yes $>$. Acesso em: 9 nov. 2011

DAMIANO, D. L.; MARTELLOTA, T. L.;

QUINLIVAN, J.; JEFFREY, M.; ABEL, M. F.

Deficits in eccentric versus concentric torque in children with spastic cerebral palsy. Medicine \& Science in Sports \& Exercise, Madison, v. 33, p. 117-122, 2001. Disponível em:

$<$ http://journals.lww.com/acsm-

msse/Abstract/2001/01000/Deficits in eccentric versus concentric torque in.18.aspx $>$. Acesso em: 9 nov. 2011

DAMIANO, D. L.; QUINLIVAN, J. M.; OWEN, B. F.; PAYNE, P.; NELSON, K. C.; ABEL, M. F. What does the Asworth scale really measure and are instrumented measures more valid and precise? Developmental Medicine \& Child Neurology, London, v. 44, p. 112-118, 2002. Disponível em: <http://dx.doi.org/10.1017/S0012162201001761>. Acesso em: 9 nov. 2011

DAMIANO, D. L.; ABEL, M.; ROMNESS, M.; OEFFINGER, D.; TYLKOWSKI, C.; GORTON, G.; BAGLEY, A.; NICHOLSON, D.; BARNES, D.; CALMES, J.; KRYSCIO, R.; ROGERS, S. Comparing functional profiles of children with hemiplegic and diplegic cerebral palsy in GMFCS Levels I and II: are separate classifications needed. Developmental Medicine \& Child Neurology, London, v. 48, p. 797-803, 2006. Disponível em: <http://dx.doi.org/10.1017/S0012162206001733>. Acesso em: 9 nov. 2011

DROUIN, J. M.; VALOVICH-MCLEOD, T. C.; SHULTZ, S. J.; GANSNEDER, B. M.; PERRIN D.H. Reliability and validity of the Biodex system 3 pro isokinetic dynamometer velocity, torque an position measurements. European Journal of Applied Physiology, Berlin, v. 91, p. 22-29, 2004. Disponível em: <http://dx.doi.org/10.1007/s00421003-0933-0>. Acesso em: 9 nov. 2011

DURIGON, O. F. S.; SÁ, C. S. S.; SITTA, L. V. Validação de um protocolo de avaliação do tono 
muscular e atividades funcionais para crianças com paralisia cerebral. Revista Neurociências, São Paulo, v. 12, p. 87-93, 2004. Disponível em: $<$ http://www.unifesp.br/dneuro/neurociencias/Neur ociencias12-2.pdf\#page=30 >. Acesso em: 9 nov. 2011

ENGSBERG, J. R.; OLREE, K. S.; ROSS, S. A.; PARK, T. Quantitative clinical measure of spasticity in children with cerebral palsy. Archives of Physical Medicine \& Rehabilitation, Chicago, v. 77, p. 594-599, 1996. Disponível em: $<$ http://dx.doi.org/10.1016/S0003-9993(96)903019 $>$. Acesso em: 9 nov. 2011

LANFREDI, A. P. A.; NARVARES, S.; PERES, P. T.; MATTA, D. S. P. Nível de habilidade motora e funcional em crianças com PC. Reabilitar, São Paulo, v. 25, p. 20-23, 2004. Disponível em: $<$ http://bases.bireme.br/cgibin/wxislind.exe/iah/online/? IsisScript=iah/iah.xis\& $\underline{\mathrm{src}=\text { google \&base }=\text { ADOLEC\&lang }=\text { p\&nextAction }=1}$ nk\&exprSearch=413817\&indexSearch=ID>. Acesso em: 9 nov. 2011

MANCINI, M. C.; FIÚZA, P. M.; REBELO, J. M.; MAGALHÃES, L. C.; COELHO, Z. A. C.; PAIXÃO, M. L.; GONTIJO, A. P. B.; FONSECA, S. T. Comparação do desempenho de atividades funcionais em crianças com desenvolvimento normal e crianças com paralisia cerebral.

Arquivos de Neuro-Psiquiatria, São Paulo, v. 60, p. 446-452, 2002. Disponível em: $<$ http://dx.doi.org/10.1590/S0004282X2002000300020>. Acesso em: 9 nov. 2011

MILLER, G. Paralisias cerebrais: uma visão geral. In: MILLER, G.; CLARK, G. D. Paralisias cerebrais: causas, consequências e conduta. São Paulo: Manole, 2002. p. 1-41.

MOREAU, N. G.; LI, L.; GEAGHAN, J. P.; DAMIANO, D. L. Fatigue resistance during a voluntary performance task is associated with lower levels of mobility in cerebral palsy. Archives of Physical Medicine \& Rehabilitation, Chicago, v. 89, p. 2011-2016, 2008. Disponível em: <http://dx.doi.org/10.1016/j.apmr.2008.03.012>. Acesso em: 9 nov. 2011

ROSS, A. S.; ENGSBERG, J. R. Relation between spasticity and strength in individuals with spastic diplegic cerebral palsy. Developmental Medicine \& Child Neurology, London, v. 44, p. 144-157, 2002. Disponível em: <http://dx.doi.org/10.1017/S0012162201001852>. Acesso em: 9 nov. 2011

SCHNEIDER, P.; RODRIGUES, L. A.; MEYER, F. Dinamometria computadorizada como metodologia de avaliação da força muscular de meninos e meninas em diferentes estágios de maturidade. Revista Paulista de Educação
Física, São Paulo, v. 16, p. 35-42, 2002.

Disponível em:

$<$ http://www.usp.br/eef/rpef/v16n12002/v16n1p35. pdf >. Acesso em: 9 nov. 2011

SCHOLTES, V. A.; DALLMEIJERI, A. J.; RAMECKERS, E. A.; VERSCHUREN, O.; TEMPELAARS, E. Lower limb strength training in children with cerebral palsy: a randomized controlled trial protocol for functional strength training based on progressive resistance exercise principles. BMC Pediatrics, London, v. 8, p. 41, 2008. Disponível em:

<http://dx.doi.org/10.1186/1471-2431-8-41>. Acesso em: 9 nov. 2011

SHUMWAY-COOK, A.; WOOLLACOTT, M. H. Controle Motor: teoria e aplicações práticas. 2. ed. Barueri: Manole, 2003.

TUZSON, A. E.; GRANATA, K. P.; ABEL, M. F. Spastic velocity threshold constrains functional performance in cerebral palsy. Archives of Physical Medicine \& Rehabilitation, Chicago, v. 84, p. 1363-1368, 2003. Disponível em: $<$ http://www.ncbi.nlm.nih.gov/pmc/articles/PMC17 90963/pdf/nihms-12863.pdf >. Acesso em: 9 nov. 2011

\section{Endereço:}

A/C Coordenadoria de Fisioterapia

Patrícia Martins Franciulli

Rua Taquari, 546, Mooca

03166-000

São Paulo SP Brasil

551127991944

E-mail: franciulli@usp.br

Recebido em: 13 de junho de 2010. Aceito em: 22 de novembro de 2011.

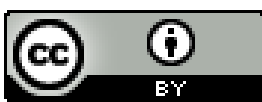

Motriz. Revista de Educação Física. UNESP, Rio Claro, SP, Brasil - elSSN: 1980-6574 - está licenciada sob Creative Commons - Atribuição 3.0 